\title{
Preliminary evaluation of supervised discharge order in the South and West Region
}

\author{
Damian Mohan, Chris Thompson and Mark A. Mullee
}

\begin{abstract}
Six months after the introduction of the Mental Health (Patients in the Community) Act 1995, this study shows that the new legistation has been implemented in its first six months of existence. Lack of resources and increased papenwork are identfiled by some doctors as reasons for their reluctance to proceed with implementation of supervised discharge. Patients who were placed on supervised discharge were found to be mostly young, mentally ill patients previously detained under Section 3 of the Mental Health Act 1983, as yet, the 1995 Act does not appear to have been implemented in the after care of 'forensic' patients or those with mental impairment.
\end{abstract}

The Mental Health (Patients in the Community) Act 1995 became effective from 1 April 1996. The prospect of using supervised discharge as a legal framework to assist in managing mentally ill patients in a community setting stimulated much debate in the medical literature (Bluglass 1993a,b; Burns et al, 1993; Eastman, 1994). The Act amended the Mental Health Act 1983, by providing for after care under supervision, also known as a supervised discharge order. This changed the law for some 'sectioned' patients being discharged from hospital. The patient's responsible medical officer (RMO) can now make an application to the health authority which becomes legally responsible, along with the local authority, for the patient's after care. If the application is accepted the patient is placed on a supervised discharge order when their Section 3 expires and they leave hospital. Patients who are already living in the community on Section 17 leave from a Section 3 admission, and patients detained under Part III of the Act without restriction orders are also eligible for the supervised discharge order.

In all cases the RMO must show that the patient would benefit from the supervised discharge order by being enabled to receive the services they need. Many psychiatrists have expressed the opinion that the inadequacy of their services would make all their patients ineligible under this clause alone. In addition doubts have been expressed about the usefulness of an order which allows the mental health team to compel a patient to live in a particular place and to attend for treatment but does not compel them to have that treatment. Furthermore the new provision which allows a 'supervisor' or a 'community RMO' (two new legally defined terms) to convey a patient to the place where they may be offered (but not compelled) to take treatment, has concerned many as an unwarranted intrusion on civil liberties with inadequate compensatory benefit. Previously the only community-based order was guardianship, which was used very seldom. For all these reasons there has been doubt about whether the new order will be used and, if so, whether it will be helpful to patients themselves.

We therefore wished to review the use of the new provisions at an early stage of their implementation, to make an assessment of the frequency of use, training requirements of doctors and perceived usefulness in patient care.

\section{The study}

Local ethics committee approval was obtained for a postal survey which was carried out using a structured questionnaire. This sought information about the doctors' demographic characteristics, whether they had patients on section under their care (and were therefore eligible to use the supervised discharge order), whether they had considered using the supervised discharge order and if so, details of the actual implementation, or reasons why it had eventually not been implemented. It also asked respondents about training, knowledge and attitudes towards the Act.

An up-to-date register was compiled by the lead health authority for the South and West Region (Southampton and South West Hampshire) with the addresses of the 453 doctors who were approved under section 12(2) of the Mental Health Act 1983. Retired doctors and those in private practice were excluded. In October 1996. 
six months after the Act was introduced, questionnaires were sent to the following section 12(2) approved categories: psychiatrists, general practitioners, forensic examiners (police surgeons) and prison medical officers. Non-respondents received a further questionnaire two months later. Respondents who indicated that they had used a supervised discharge order were contacted by telephone to verify the information given.

Data were analysed using the statistical package SPSS version 6.1.3 (Norusis, 1990).

\section{Findings}

A total of $282(62 \%)$ responded. Fifteen were inappropriate for the survey due to long-term illness or recent retirement, leaving a total of 267 to be included in the study.

They were divided into two categories: (a) 115 consultant psychiatrists who were responsible for at least one patient detained under Sections 3 or Part III (Sections 37, 47 or 48) of the Mental Health Act 1983, thereby making them eligible to consider an application for a supervised discharge order and (b) the remaining 152 doctors who were not eligible to be an RMO under the 1995 Act since they had no patients to whom the supervised discharge order would apply.

The results presented are for those 115 consultant psychiatrists who were eligible to implement the supervised discharge order.

\section{Implementation}

Of the eligible consultants, $54(47 \%)$ had given consideration to using the supervised discharge order in at least one case. However, only $15(13 \%)$ had actually implemented it, all 15 consultants had only one patient each placed under supervised discharge.

The 39 consultants who had considered but rejected use of the supervised discharge order, were asked to identify reasons for their reluctance to proceed. The most commonly reported reason was a lack of resources ( 11 consultants), followed by increased paperwork (8), concern about litigation (5), use becoming unnecessary because the patient recovered (3) and not knowing enough to be confident in its use (2). One consultant expressed concern about mental health review tribunals censuring his decision.

There were no significant differences in the median years of psychiatric experience between the three groups: consultants who did not consider using the supervised discharge order ( $n=61$, median 15 years, interquartile range 12 20.5), consultants who considered but did not use it ( $n=37$, median 18 years, interquartile range 14.5-21 years) and those who actually used it ( $n=15$, median 16 years, interquartile range 11-27 years).

Nor was there a significant difference in their exposure to training in the use of the supervised discharge order. Ten out $15(67 \%)$ who used the Act had attended a training course compared to 57 out 100 who were eligible but had not used the Act $\left(\chi^{2}=0.50\right.$, d.f. $=1, P=0.48,95 \%$ CI for difference in proportions $=-16$ to $35 \%$ ).

A multiple choice questionnaire showed a slight difference in knowledge between users when compared with those who were eligible but did not utilise the Act. The median score was six (interquartile range 4-6) out of a possible eight for consultants who utilised the Act. Eligible non-users, however, scored a median of five (interquartile range 3-6). This difference failed to reach statistical significance (MannWhitney $U=562, P=0.12$ ).

The eligible consultants, who had used the supervised discharge order (15), were asked if they felt that the new legislation had helped or hindered them in the management of patients. Of those who gave a valid response, $8(62 \%)$ said they felt that the legislation had helped them and $12(92 \%)$ that it had at least not hindered them in the management of patients.

The attitudes towards the supervised discharge order among consultants who had implemented it (15) were divided. When asked if the use of supervised discharge order had altered their general opinion, four had become more negative about it and five more positive. Four remained concerned about the principle of informed consent in relation to the order. Seven felt civil liberties were not a relevant issue, while four remained concerned about them. Six felt it did give the psychiatrist significantly more powers and equally, five disagreed with this. Although six felt that it did help to maintain patients in the community, 10 did not feel that it facilitated discharge. While two felt they would be more likely to use guardianship in the future, nine did not.

\section{Characteristics of patients}

In the South and West Region the 15 consultants who utilised the 1995 Act reported on 15 patients who had been placed on supervised discharge. Of these all had been on Section 3 , five of whom had been on Section 17 leave (Figure 1). No patients had previously been detained under Part III of the Mental Health Act 1983.

Thirteen patients were classified as having a mental illness and two patients belonged to the psychopathic disorder category. Only two consultants had used the act for patients over 65 years old. 


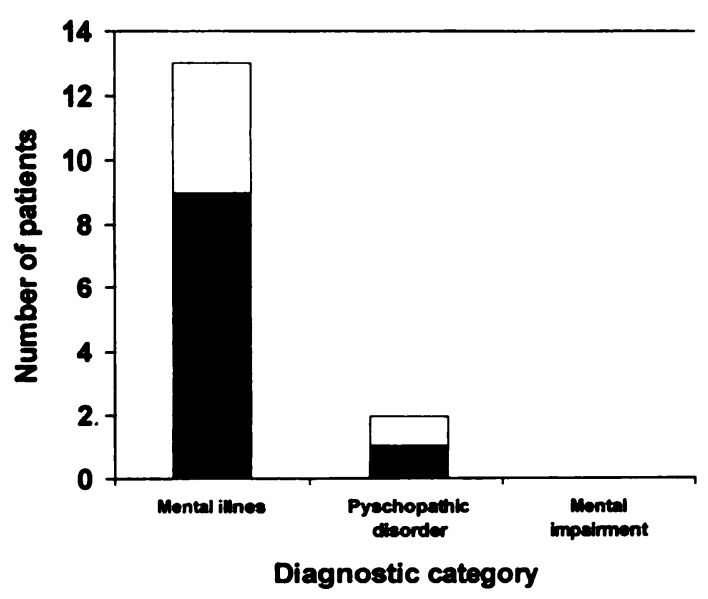

Figure 1. Diagnostic category of patients placed under supervised discharge by 15 consultants. $\square$, Section 3: $\square$, Section 3 and 17

\section{Comments}

We were surprised that in the first six months of the existence of the supervised discharge order $47 \%$ of eligible doctors in the South and West Region who responded to our questionnaire had at least considered using it and $13 \%$ had made at least one patient subject to the order. Lack of resources was the single most likely reason for not using it, which is not surprising since one of the criteria for application is that the order must be likely to help the patient to gain access to the resources needed. The paper work, which is extensive, was also seen as a disincentive.

Before the Act was implemented some felt that it would apply particularly to the elderly but in the first six months it has been used mainly for younger patients with mental illness, with only two consultants using it for patients over 65. Six months after its introduction only $58 \%$ of consultants who might have used the Act had received any training in it, and rather alarmingly this was unrelated, as was knowledge of the order, to whether or not it had been used by the consultant.
This study was neither designed to collect information from the patients' or relatives' perspective, nor was there an independent follow up of clinical outcome in the group on the order and a comparison group. Now that it is clear that the Act will be used (or that it will at least have a vogue among some consultants) further studies will be necessary to assess more adequately the therapeutic value of these new powers.

\section{Acknowledgement}

We are grateful to David Carson, Senior Lecturer in Law, University of Southampton for comments on the questionnaire design.

\section{References}

BLUGLASS R. (1993a) Maintaining the treatment of mentally ill people in the community. British Medical Joumal. 306. $159-160$.

- (1993b) New powers of supervised discharge of mentally ill people. British Medical Journal, 307, 1160

Burns, T., GODDARD, K. \& BALE, R. (1993) Mental health professionals favour community supervision orders. British Medical Journal, 307, 803.

EASTMAN, N. (1994) Mental health law: Civil liberties and the principle of reciprocity. British Medical Journal, 308 43-45.

NoRUSIS, M. J. (1990) The SPSS Guide to Data Analysis for Release 4. Chicago, IL: SPSS Inc.

-Damian Mohan, Lecturer in Forensic Psychiatry, Professorial Unit, Broadmoor Hospital and University Department of Mental Health, Royal South Hants Hospital, Brintons Terrace, Southampton SO14 OYG; Chris Thompson, Professor of Psychiatry, University Department of Mental Health, Royal South Hants Hospital, Brintons Terrace, Southampton SO14 OYG; and Mark Mullee, Senior Research Fellow, Medical Statistics and Computing, University of Southampton, Level B, South Academic Block, Southampton General Hospital, Southampton SO16 6YD.

*Correspondence 